\title{
Pediatric neuroimaging in early childhood and infancy: challenges and practical guidelines
}

\section{Citation}

Raschle, Nora, Jennifer Zuk, Silvia Ortiz-Mantilla, Danielle D. Sliva, Angela Franceschi, P. Ellen Grant, April A. Benasich, and Nadine Gaab. 2012. "Pediatric Neuroimaging in Early Childhood and Infancy: Challenges and Practical Guidelines." Annals of the New York Academy of Sciences 1252 (1) (April): 43-50. doi:10.1111/j.1749-6632.2012.06457.x.

\section{Published Version}

doi:10.1016/j.neuroimage.2010.05.075

\section{Permanent link}

http://nrs.harvard.edu/urn-3:HUL.InstRepos:30085110

\section{Terms of Use}

This article was downloaded from Harvard University's DASH repository, and is made available under the terms and conditions applicable to Other Posted Material, as set forth at http:// nrs.harvard.edu/urn-3:HUL.InstRepos:dash.current.terms-of-use\#LAA

\section{Share Your Story}

The Harvard community has made this article openly available.

Please share how this access benefits you. Submit a story.

\section{Accessibility}




\title{
Pediatric neuroimaging in early childhood and infancy: challenges and practical guidelines
}

\author{
Nora Raschle ${ }^{1,2}$, Jennifer Zuk ${ }^{1}$, Silvia Ortiz-Mantilla ${ }^{3}$, Danielle D. Sliva ${ }^{4}$, Angela \\ Franceschi $^{5}$, P. Ellen Grant ${ }^{2,4,5,6}$, April A. Benasich ${ }^{3}$, and Nadine Gaab ${ }^{1,2,7}$ \\ ${ }^{1}$ Laboratories of Cognitive Neuroscience, Division of Developmental Medicine, Children's Hospital \\ Boston, Boston, Massachusetts \\ ${ }^{2}$ Harvard Medical School, Boston, Massachusetts \\ ${ }^{3}$ Center for Molecular and Behavioral Neuroscience, Rutgers, The State University of New \\ Jersey, Newark, New Jersey \\ ${ }^{4}$ Fetal-Neonatal Neuroimaging and Developmental Science Center, Children's Hospital Boston, \\ Boston, Massachusetts \\ ${ }^{5}$ Department of Radiology, Division of Neuroradiology, Children's Hospital Boston, Boston, \\ Massachusetts \\ ${ }^{6}$ Department of Medicine, Division of Newborn Medicine, Children's Hospital Boston, Boston, \\ Massachusetts \\ ${ }^{7}$ Harvard Graduate School of Education, Boston, Massachusetts
}

\section{Abstract}

\begin{abstract}
Structural and functional magnetic resonance imaging (fMRI) has been used increasingly to investigate typical and atypical brain development. However, in contrast to studies in school-aged children and adults, MRI research in young pediatric age groups is less common. Practical and technical challenges occur when imaging infants and children, which presents clinicians and research teams with a unique set of problems. These include procedural difficulties (e.g., participant anxiety or movement restrictions), technical obstacles (e.g., availability of childappropriate equipment or pediatric MR head coils), and the challenge of choosing the most appropriate analysis methods for pediatric imaging data. Here, we summarize and review pediatric imaging and analysis tools and present neuroimaging protocols for young nonsedated children and
\end{abstract}

\section{(C) 2012 New York Academy of Sciences.}

Address for correspondence: Nadine Gaab, Ph.D., Children's Hospital, Boston, Department of Medicine, Division of Developmental Medicine, Laboratories of Cognitive Neuroscience, 1 Autumn Street, Mailbox \# 713, Boston, MA 02115.

nadine.gaab@childrens.harvard.edu.

Conflicts of interest

The authors declare no conflicts of interest.

Supporting information

Additional supporting information may be found in the online version of this article:

Figure S1. Overview of original research (OR) and review articles (RA) that addresses the issues of pediatric imaging and/or provide techniques for working with children within the imaging environment; in chronological order.

Figure S2. Overview of original research (OR) and review articles (RA) that addresses the issues of pediatric imaging and/or provide techniques for working with infants within the imaging environment; in chronological order.

Figure S3. Summary of the success rate of imaging infants and young children within our laboratories. Note that a subset required multiple attempts (number of imaging sessions) to successfully acquire the images. Multiple sessions markedly increased the success rate.

Please note: Wiley-Blackwell is not responsible for the content or functionality of any supporting materials supplied by the authors. Any queries (other than missing material) should be directed to the corresponding author for the article. 
infants, including guidelines and procedures that have been successfully implemented in research protocols across several research sites.

\section{Keywords}

pediatric; imaging; children; magnetic resonance imaging; fMRI; MRI

\section{Introduction}

The advent of magnetic resonance imaging (MRI) has opened up new possibilities for studying human brain structure and function across the lifespan. An increase in the use of structural and functional MRI (fMRI) in infants and young children can further add to our understanding of brain development. For example, MRI research has revealed differences in brain structure and function in individuals with disabilities (e.g., dyslexia ${ }^{1-3}$ ) compared with typical controls, and working with infants and young children may unveil the developmental trajectory of such disabilities. Neuroimaging young children additionally allows for the investigation of brain plasticity during this rapid developmental period and can potentially reveal how certain perceptual, procedural, and cognitive skills, such as music perception and musical skills, develop. ${ }^{4}$ However, in contrast to studies in school-aged children and adults, MRI research in young pediatric age groups is less common. ${ }^{5}$ Studies involving children under the age of 6 are particularly rare, given the practical and technical challenges involved (e.g., Refs. 5, 6). Practical challenges of pediatric neuroimaging sessions include procedural difficulties (e.g., participants', anxiety or motivation, movement restriction, putting an infant to sleep in an unfamiliar environment, and parent's anxiety), technical obstacles (e.g., availability of child-appropriate equipment, masking and attenuation of scanner background noise [SBN]), as well as the challenge of choosing the most appropriate analysis methods (e.g., pediatric brain templates and adequate movement detection tools). In clinical populations, MRIs of infants and children are routinely obtained under sedation, ${ }^{7-9}$ which eliminates a subset of these challenges. However, for ethical reasons, sedation is not an option for most developmental neuroimaging research. Furthermore, there is a strong push from clinicians and hospital administrators to reduce the overall need for sedation and anesthesia for cost containment and more importantly to prevent any potential negative sequelae, particularly in those receiving multiple MRI studies.

Several methods have been developed to improve an infant or child's compliance during neuroimaging sessions within the clinical (e.g., Refs. 10-12) or research setting (e.g., Refs. $13,14)$. General approaches for imaging young children include play therapy, ${ }^{14}$ behavioral training ${ }^{10-12,15-17}$ and simulation, ${ }^{18}$ the use of mock scanner areas, ${ }^{13-19}$ basic relaxation, ${ }^{20}$ and a combination of these techniques. ${ }^{21}$ The most common practices for nonse-dated newborns and infants are the natural sleep technique $22-25$ and the feed and wrap procedure. ${ }^{26,27}$ Figures S1 and S2 provide a literature overview of published protocols, guidelines, and empirical research studies using pediatric neuroimaging protocols and their sample size and success rate.

This paper summarizes successful methods for applying guidelines and recommendations on how to successfully perform neuroimaging studies in nonsedated infants and young children. Furthermore, strategies for overcoming experimental and analysis limitations and ethical implications of neuroimaging in pediatric populations are discussed.

\section{General considerations}

The age of a pediatric participant taking part in neuroimaging research should strongly influence the research protocol for pediatric imaging. Preschool-aged children may 
participate in structural and functional neuroimaging studies, in which the child is required to be awake and alert while performing a certain perceptual or cognitive task. Infants are usually enrolled in studies of brain structure or resting state fMRI, which are performed while the participants are asleep. fMRI techniques have been successfully applied in awake infants, but report a very high attrition rate. ${ }^{28,29}$ Although the focus in working with preschool-aged children or older mostly lies on the child itself, the caregiver-child interaction becomes just as important during infant neuroimaging. Overall, clear communication and a child-centered, age-appropriate approaches are the fundamental aspects of pediatric neuroimaging.

\section{Terminology}

When interacting with participating children and families, it is imperative to use positive, child-friendly terminology that can be easily understood. A session may get started with "Do you know what will happen today?" All equipment should be labeled in a child-appropriate way: for example, the MRI machine may be called "brain camera," the scanner noise "camera click," and the head coil "mirror holder." Phrases like "It is really loud but won't hurt you" or "Are you doing okay inside the machine?" should be avoided. Furthermore, careful communication with all family members present during the session is just as important, if not more important, than the communication with the participants themselves to avoid parental/caregiver anxiety, which in turn impacts the behavior of the young child or infant. This involves giving the parents a clear outline of the neuroimaging session, describing each step and tool involved, reviewing safety considerations, and clarifying the research team's goal. When imaging infants, potential stress may be avoided by telling parents upfront that it is not unusual for a child to fail to fall asleep on the first visit or to wake up before the protocol is completed. A website describing each protocol step, tool, and challenge can help with the preparation process for any age group.

\section{Environment}

Scheduling an initial introductory meeting before the first neuroimaging session can help diminish both parent and child anxiety by familiarizing them with the research team and setting. Photographs, and/or a brief video overview of an actual session (either via web pages or brochures) may be combined with a tour of the actual MRI area or the mock scanner environment. Research teams working with preschoolers and older children especially benefit from the use of a mock scanner environment before or on the day of testing. It enables the research team to demonstrate the actual imaging session and may include a mock scanner with a move-able scanner bed, head coil, response tools, mirror and video system, and integrated MR sounds. Some facilities even incorporate a feedback system as part of the mock scanner area, allowing for the observation of movement and appropriate feedback to train children to lie still (e.g., Refs. 10, 17). Most importantly, the mock scanner area provides a child-friendly, appropriate preview of the actual neuroimaging session, and permits the research team to adopt a playful approach to an otherwise strictly medical topic. In addition, research teams are encouraged to have child-appropriate toys, child-sized table and chairs, snacks and drinks, and to invite participants to bring family and friends to increase children's comfort and motivation. When imaging infants, the mock scanner area is used mainly to familiarize the parents with the scanner equipment and the MRI sounds. Mock preparation further offers an opportunity to develop a relationship between the infant and the researcher, and can be used to recreate individual napping/bedtime routines within the MRI environment.

\section{Affective state}

It is crucial to recognize feelings of anxiety, frustration, or boredom and address potential issues in a child-friendly manner because children are not always able to express emotions 
directly. Gauging parent comfort and the level of continued consent throughout the process is equally important, particularly during neuroimaging of infants. The parent's comfort level directly influences the infant's feelings of well-being and contentment, which is critical during the natural sleep technique. Given their expertise in child development and extensive experience with children/families in the hospital environment, a child life specialist (CLS) can be an integral part of the multidisciplinary pediatric neuroimaging team.

\section{MRI equipment}

Depending on the scope of the pediatric neuroimaging session, different MRI-compatible tools may be needed. This section highlights some examples of equipment involved during neuroimaging sessions with infants and children.

Response buttons-Response tools used during the neuroimaging session should be ageappropriate in size and shape. It is recommended to position them comfortably to diminish motion when the participant attempts to reach or play with them (e.g., placing child-friendly buttons an arm-length from the participant works well).

Audio system-The frequency of sounds emitted during MR image acquisition ranges from $0-9,000 \mathrm{~Hz}^{30}$ at intensity levels up to $115 \mathrm{~dB}$ with 3 tesla. ${ }^{30}$ When working with young children who are participating in an auditory research experiment, child-friendly ear buds and/or child-sized headphones can mask the majority of SBN while allowing for the presentation of auditory stimuli. When working with infants, the goal is to decrease SBN as much as possible to avoid sleep disruption or startle. The noise can be reduced by $20-30 \mathrm{~dB}$ with the use of foam earplugs or industrial-grade earmuffs. ${ }^{31}$ In addition, music or the infant's favorite lullabies can be played to mask some of the variations in the scanner noise. A steady-state sound can be an aid to sleep; however, noise that abruptly changes in sound level or intensity in a nonprogressive manner is much more likely to result in arousal and waking.

Motion attenuation-A foam mattress to line the scanner bed reduces the amount and intensity of vibration, additionally increasing comfort. For sleeping infants, a foam helmet can help reduce bone conductance, which adds sound attenuation of about $6 \mathrm{~dB}$, reduces vibration, and provides additional stability.

Video/video-goggle system-For imaging older children, a video system can be used to present experimental stimuli or movies during image acquisition. However, younger children may be frightened by a goggle system, so a traditional projector-screen combination may be used.

\section{Experimental design and image acquisition}

Pediatric imaging for research purposes presents many challenges during both image acquisition and data analysis. Challenges may begin long before the first image is taken, in fact as early as during the conception and design of the research.

\section{Experimental design}

Young children often have difficulties switching between two different task instructions; however, task switching is frequently required during a traditional block or event-related design in fMRI. Our experience has shown that, if possible, it is advisable to use a block design and to separate task conditions (e.g., experimental task and control task) into two separate runs to avoid confusion. 
Furthermore, the overall session length should be as short as possible and not be any longer than 90 min for a preschooler (which includes short 5-6 minute runs with breaks between each run and a total maximum of 30-40 min of actual scanning). In addition, the intense SBN, generated by the shifting of gradient coils during conventional continuous image acquisition, is one potential cause for child anxiety or discomfort. SBN affects auditory stimuli delivery, ${ }^{32}$ leads to activation in auditory/language areas, ${ }^{33,34}$ leads to differences in attentional demands, ${ }^{35}$ and also influences the default mode. ${ }^{36}$ Inhibiting the SBN during a cognitive demanding task can be quite difficult for a young child and/or a clinical population, and this should be taken into account when comparing different age groups or clinical and nonclinical populations. One way to circumvent exposure to the SBN is to use interleaved data acquisition designs, such as sparse temporal sampling, ${ }^{33,34,37}$ if time permits.

\section{Image acquisition}

There are many challenges during image acquisition and analysis of pediatric imaging data. These barriers are most prevalent in the first three years of life, but are also present in older children and may include challenges relating to design limitations (e.g., constrained session duration in children, leading to a decrease in statistical power; and difficulty in designing task-based fMRI for infants because MR studies are most successful when the infant is imaged asleep). Challenges may also include the presence of increased movement-related artifacts or differences based on distinct anatomy (e.g., difficulties in obtaining MR head coils that provide similar fit across age, differences in brain shape and size across age, differences in brain contrast, baseline diffusivity, baseline blood flow and regional regulation, the shape of the hemodynamic response function, or default mode of brain function across age).

Many research groups are currently working on finding solutions for these challenges. For example, many collaborative groups including ours combine approaches from industry and child-life professionals in the hospital setting and further work on optimizing techniques. In parallel, technical groups are developing prospective motion mitigated sequences, and MRI coils tailored to the head size of the subject are also under development. ${ }^{38,39}$ Development of novel pulse sequences that exploit high density-phased array coils to increase the speed of image acquisition have the potential to further increase success. Pediatric brain templates are becoming available to improve data analysis and to help account for age-related changes (e.g., Refs. 8,40, 41). Furthermore, several research labs have developed techniques to better align and normalize brains that differ in size and shape. For instance, surface-based registration has been shown to provide significantly better alignment across different age groups. ${ }^{42}$ However, these templates do not allow capture of the full range of information available, and therefore multiple sites are working on " $4 \mathrm{D}$ " atlases that provide information on maturation of substructures. ${ }^{43}$

Overall, much is still unknown about the biological differences between the immature and mature brain that could influence data analysis. At each step along the way, the fundamental assumptions for any data analysis tool need to be questioned to be sure these assumptions still hold for the age of infant or child participant. As a result, a close collaboration between technical and developmental experts is needed as we continue to study these younger age groups.

\section{Preparation and neuroimaging procedures for infants and children}

\section{Neuroimaging infants}

Obtaining detailed information about the child's nap and feeding schedule is helpful in facilitating the preparation for an infant session and for deciding the best time to schedule 
the MRI. For some babies, and always in accordance with the parent's wishes, an earlier nap may be omitted so that the infant is sleepy upon arrival. Completing several interesting, but not too stimulating, tasks immediately before the scan can further fatigue the child. Parents are encouraged to bring anything that the infant may need for his or her bedtime routine (a familiar blanket, favorite toy, or lullaby music). Listening to a CD containing scanner background sounds (optionally overlaid with favorite lullabies) before the day of the neuroimaging session will prepare the infant for the upcoming procedure. Exposure to the scanner sounds should ideally be started at least a week before imaging. Encouraging frequent exposure to the sounds (e.g., during both awake and nap times) increases the habituation effect and may decrease startle responses during neuroimaging.

When scanning infants, replicating normal bedtime routines in the imaging suite, including bathing, feeding, dimming the lights, and playing soft lullaby music, has proven to be effective. ${ }^{22}$ Offering a rocking chair, portacrib, bathtub, or blankets for swaddling may help parents to put the baby to sleep. Playing a CD of the MRI sequences in the room where the infant falls asleep provides a stable auditory environment throughout the process. To ease the transition between a nursery-type environment and the MRI suite, an alternative approach is to have the infant fall asleep inside the MRI suite using a MRI-compatible (inflatable) crib. Some caregivers may decide to perform their sleep routine (e.g., nursing, lullabies, etc.) inside the MRI suite or even on the MRI scanner bed. The caregiver should be allowed to try to put their child to sleep, as long as they are comfortable. The research team should leave them on their own if at all possible and not "hover." Reserving at least a two-hour time slot with the MRI allows for flexibility.

Once the infant has fallen asleep, earplugs, ear-muffs ("Minimuffs," Natus Medical Inc., San Carlos, CA), and a sound attenuation helmet can be placed on the infant. ${ }^{24,25}$ Earplugs should be placed after feeding, as having the ears blocked while nursing can make the infants uncomfortable. The infant can be slowly and gently lied on the prepared scanner bed, which should be ideally covered with a well-secured visco-elastic foam mattress. Laying a warm blanket on top of the mattress can alleviate the cool temperature in the MRI suite. The infant's head should then be positioned into the coil and the researcher can then carefully adjust the restraint straps in place to secure the infant and limit his or her movements. Once the infant has been snuggled into the space with a soft blanket and sleeps soundly, the scan can proceed.

Throughout the scan, the caregiver and a team member should stay in the imaging suite. If the infant begins to wake, MRI acquisition can be paused and an attempt made to soothe the infant back to sleep. If the infant cries or becomes distressed at any time during the scan, it is advisable to stop and immediately remove the baby from the scanner. Regardless of the success or failure of image acquisition, offering toys and onesies to infants are a nice token of appreciation and after a success, providing a $\mathrm{CD}$ with images of the infant brain to take home is an incentive for parents.

\section{Neuroimaging young children}

Presenting young children with a cognitive task to complete during functional imaging requires the adaptation of the tasks, instructions, and incentives used. A child-friendly theme can be useful in guiding a young child through the training and actual neuroimaging session. For example, an adventure story can motivate the child to finish all of the tasks requested. Short movies can be useful to lead into the session, engage the child, and reduce any initial anxiety. Additionally, virtual sticker charts can be used to provide feedback about the progress of the neuroimaging session and to motivate the child to complete as many sequences as possible (see also Ref. 44). 
Children 4 years and older are best prepared for the fMRI session in a mock scanner area. The scope of the neuroimaging session can be explained to participants and families using applied examples and the material used during actual imaging (e.g., button boxes and headphones) can be revisited in a safe way. Once a general introduction to the MRI has been provided, the child can listen to instructions for the task and then practice while sitting on the mock scanner bed. After the child understands the task, the researcher can gradually add the use of headphones, present some prerecorded scanner sounds in the background, and have the child lie down in the mock scanner bed while simultaneously practicing the training task to replicate the actual MRI experience. If the child cannot distinguish between left and right, a response button may be labeled "monkey button" instead of "right button" and a toy animal can be placed on the appropriate side as a reminder.

One major challenge of working with young children during functional tasks is keeping movements to a minimum during image acquisition. It is advisable to discuss the impact of motion on the brain images with the child. A digital camera may be used to demonstrate the impact of movement on image quality. Print-outs of sharp and blurry pictures (e.g., of animals) or playing games, such as the game of "freeze" (challenge the child to stay still as long as possible), can be used for illustration purposes. It is best to remind the child not to speak during image acquisition because this can cause reduced task performance and data quality. Another helpful strategy is to train the child that a gentle hand press on their leg indicates there is too much movement. A member of the research team should stay in the MRI room throughout the session with the child and should implement the "hand on leg" procedure if the child demonstrates significant movement, which serves as a means of communication between the research team and the child during the experiment.

If a mock scanner is not available, game strategies such as employing play therapy techniques ${ }^{45}$ are a recommended alternative. Providing pictures or videos of an MR scanner effectively depicts the MRI experience, ${ }^{10}$ and desensitization to the procedure by this exposure and operant training techniques help to reduce anxiety in children. ${ }^{12,17}$

Researchers should keep in mind that the transition from mock training to the actual MR also brings changes to the environment (fewer toys, more medical personnel and supplies, change in temperature, etc). Thus, it is important to be attentive to the child's reaction upon entering the room and respond accordingly. Allowing the parent to accompany the child and offering comfort items may effectively smooth the transition. If a child displays resistance to any aspect of the MRI experiment, address the concerns immediately and carefully. Offering breaks during the neuroimaging session can be helpful. The researcher should take all the time necessary to make the child comfortable with the MR environment. Upon completion of image acquisition, offering incentives and a $\mathrm{CD}$ with images of the brain to the child is a nice reward for participation.

\section{Ethical implication and conclusion}

Here, we provide applied guidelines and recommendations on how to successfully perform neuroimaging studies on nonsedated infants and young children. These techniques have proven to be successful in a large group of infants and young children across several research sites (see Fig. S3). It is important to keep in mind that along with the advent of more accessible (pediatric) neuroimaging, ethical challenges have also arisen. Although these issues have emerged and have been discussed in detail in regard to adult neuroimaging, such ethical issues are not only present when considering pediatric neuroimaging, but also may even be magnified. ${ }^{46,47}$ However, the anticipated benefits of using pediatric fMRI in nonclinical populations are considerable and may have far-reaching advantages including application to classroom settings. ${ }^{47}$ Nevertheless, researchers should 
carefully consider what method is most appropriate for their experimental question and participant age range. Thomason has highlighted the positive experiences of pediatric research participants, but such data are not available yet for younger children. ${ }^{48}$ Furthermore, Connors and Singh strongly emphasize that neuroimaging data are often misinterpreted by the general public and that there are a series of subtle ways in which neuroimaging data are and will affect children's lives such as the shaping of national health, strategies for criminal risk assessment, and educational practice and its overall implication for policy, education, and family life. ${ }^{49}$ It is also important to keep ethical considerations in mind and to frequently reevaulate advances in the field of pediatric imaging in terms of its ethical implications. This will help improve existing imaging protocols and guidelines and will also facilitate development of new technological advances that will improve comfort for the children scanned and enhance data quality.

\section{Supplementary Material}

Refer to Web version on PubMed Central for supplementary material.

\section{Acknowledgments}

The authors thank W.C. Liu and D. Johnson from UMDNJ for their invaluable help in running nonse-dated scans in infants. The authors also thank the researchers and staff at the Center for Molecular and Behavioral Neuroscience, who spent many hours designing the infant protocol and patiently acquiring scans. In particular, they thank J. Flax, S. Paterson, N. Badridze, J. Byrne, and S. Marken. The authors would also like to thank the Children's Hospital Boston MRI Team and the members of the Gaab lab who helped conduct these scans. And of course, they thank all the parents, babies, and children who worked so hard to help them acquire these important images of the developing brain. This work was supported by a grant from the Harvard Catalyst (to N.G. and E.G.) and the Santa Fe Institute Consortium (to A.A.B.), with additional funding from the Elizabeth H. Solomon Center for Neurodevelopmental Research.

\section{References}

1. Richlan F, Kronbichler M, Wimmer H. Functional abnormalities in the dyslexic brain: a quantitative meta-analysis of neuroimaging studies. Hum Brain Mapp. 2009; 30:3299-3308.10.1002/hbm.20752 [PubMed: 19288465]

2. Shaywitz S, Shaywitz B. Paying attention to reading: the neurobiology of reading and dyslexia. Dev Psychopathol. 2008; 20:1329-1349.10.1017/S0954579408000631 [PubMed: 18838044]

3. Gabrieli JD. Dyslexia: a new synergy between education and cognitive neuroscience. Science. 2009; 325:280-283. 325/5938/280 [pii]. 10.1126/science.1171999 [PubMed: 19608907]

4. Overy K, Norton A, Cronin K, et al. Examining rhythm and melody processing in young children using FMRI. Ann NY Acad Sci. 2005; 1060:210-218. 1060/1/210 [pii]. 10.1196/annals.1360.014 [PubMed: 16597768]

5. Bookheimer SY. Methodological issues in pediatric neuroimaging. Ment Retard Dev Disabil Res Rev. 2000; 6:161-165. [PubMed: 10982492]

6. Poldrack RA, Pare-Blagoev EJ, Grant PE. Pediatric functional magnetic resonance imaging: progress and challenges. Top Magn Reson Imaging. 2002; 13:61-70. [PubMed: 11847501]

7. Parazzini C, Baldoli C, Scotti G, Triulzi F. Terminal zones of myelination: MR evaluation of children aged 20-40 months. AJNR Am J Neuroradiol. 2002; 23:1669-1673. [PubMed: 12427621]

8. Altaye M, Holland SK, Wilke M, Gaser C. Infant brain probability templates for MRI segmentation and normalization. NeuroImage. 2008; 43:721-730.10.1016/j.neuroimage.2008.07.060 [PubMed: 18761410]

9. Yamada $\mathrm{H}$, et al. A milestone for normal development of the infantile brain detected by functional MRI. Neurology. 2000; 55:218-223. [PubMed: 10908895]

10. Slifer KJ. A video system to help children cooperate with motion control for radiation treatment without sedation. J Pediatr Oncol Nurs. 1996; 13:91-97. [PubMed: 8854992] 
11. Slifer KJ, Bucholtz JD, Cataldo MD. Behavioral training of motion control in young children undergoing radiation treatment without sedation. J Pediatr Oncol Nurs. 1994; 11:55-63. [PubMed: 8003262]

12. Tyc VL, Fairclough D, Fletcher B, et al. Children's distress during magnetic resonance imaging procedures. Child Health Care. 1995; 24:5-19. [PubMed: 10142086]

13. Epstein JN, et al. Assessment and prevention of head motion during imaging of patients with attention deficit hyperactivity disorder. Psychiatry Res. 2007; 155:75-82. S0925-4927(07)00012-1 [pii]. 10.1016/j.pscychresns.2006.12.009 [PubMed: 17395436]

14. Pressdee D, May L, Eastman E, Grier D. The use of play therapy in the preparation of children undergoing MR imaging. Clin Radiol. 1997; 52:945-947. [PubMed: 9413970]

15. Byars AW, et al. Practical aspects of conducting large-scale functional magnetic resonance imaging studies in children. J Child Neurol. 2002; 17:885-890. [PubMed: 12593460]

16. Slifer KJ, Cataldo MF, Cataldo MD, et al. Behavior analysis of motion control for pediatric neuroimaging. J Appl Behav Anal. 1993; 26:469-470. [PubMed: 8307831]

17. Slifer KJ, Koontz KL, Cataldo MF. Operant-contingency-based preparation of children for functional magnetic resonance imaging. J Appl Behav Anal. 2002; 35:191-194. [PubMed: 12102139]

18. Rosenberg DR, et al. Magnetic resonance imaging of children without sedation: preparation with simulation. J Am Acad Child Adolesc Psychiatry. 1997; 36:853-859. [PubMed: 9183142]

19. de Amorim e Silva CJ, Mackenzie A, Hallowell LM, et al. Practice MRI: reducing the need for sedation and general anaesthesia in children undergoing MRI. Australas Radiol. 2006; 50:319_ 323. [PubMed: 16884416]

20. Lukins R I, Davan G, Drummond PD. A cognitive behavioural approach to preventing anxiety during magnetic resonance imaging. J Behav Ther Exp Psychiatry. 1997; 28:97-104. S0005-7916(97)00006-2 [pii]. [PubMed: 9194006]

21. Hallowell LM, Stewart SE, de Amorim ESCT, Ditchfield MR. Reviewing the process of preparing children for MRI. Pediatr Radiol. 2008; 38:271-279.10.1007/s00247-007-0704-x [PubMed: 18084752]

22. Ortiz-Mantilla S, Choe MS, Flax J, et al. Associations between the size of the amygdala in infancy and language abilities during the preschool years in normally developing children. NeuroImage. 2010; 49:2791-2799. S1053-8119(09)01100-8 [pii]. 10.1016/j.neuroimage.2009.10.029 [PubMed: 19850137]

23. Liu Y, et al. Regional homogeneity, functional connectivity and imaging markers of Alzheimer's disease: a review of resting-state fMRI studies. Neuropsychologia. 2008; 46:1648-1656. [PubMed: 18346763]

24. Paterson, SJ.; Badridze, N.; Flax, JF.; Liu, WC.; Benasich, AA. A Method for Structural MRI Scanning of Non-Sedated Infants. Rutgers University; Chicago: 2004.

25. Anderson AW, et al. Neonatal auditory activation detected by functional magnetic resonance imaging. Magn Reson Imaging. 2001; 19:1-5. [PubMed: 11295339]

26. Almli CR, Rivkin MJ, McKinstry RC. The NIH MRI study of normal brain development (Objective-2): newborns, infants, toddlers, and preschoolers. NeuroImage. 2007; 35:308325.10.1016/j.neuroimage.2006.08.058 [PubMed: 17239623]

27. Gilmore JH, et al. Early postnatal development of corpus callosum and corticospinal white matter assessed with quantitative tractography. AJNR Am J Neuroradiol. 2007; 28:1789-1795.10.3174/ ajnr.A0651 [PubMed: 17923457]

28. Leroy F, et al. Early maturation of the linguistic dorsal pathway in human infants. J Neurosci. 2011; 31:1500-1506.10.1523/jneurosci.4141-10.2011 [PubMed: 21273434]

29. Dehaene-Lambertz G, et al. Language or music, mother or Mozart? Structural and environmental influences on infants' language networks. Brain Lang. 2010; 114:53-65. S0093-934X(09)00120-5 [pii]. 10.1016/j.bandl.2009.09.003 [PubMed: 19864015]

30. Hattori Y, Fukatsu H, Ishigaki T. Measurement and evaluation of the acoustic noise of a 3 Tesla MR scanner. Nagoya J Med Sci. 2007; 69:23-28. [PubMed: 17378177]

31. Zahr LK, de Traversay J. Premature infant responses to noise reduction by earmuffs: effects on behavioral and physiologic measures. J Perinatol. 1995; 15:448-455. [PubMed: 8648453] 
32. Bandettini PA, Jesmanowicz A, Van Kylen J, et al. Functional MRI of brain activation induced by scanner acoustic noise. Magn Reson Med. 1998; 39:410-416. [PubMed: 9498597]

33. Gaab N, Gabrieli JD, Glover GH. Assessing the influence of scanner background noise on auditory processing. I. An fMRI study comparing three experimental designs with varying degrees of scanner noise. Hum Brain Mapp. 2007; 28:703-720. [PubMed: 17080440]

34. Gaab N, Gabrieli JD, Glover GH. Assessing the influence of scanner background noise on auditory processing. II. An fMRI study comparing auditory processing in the absence and presence of recorded scanner noise using a sparse design. Hum Brain Mapp. 2007; 28:721-732.10.1002/hbm. 20299 [PubMed: 17089376]

35. Tomasi D, Caparelli EC, Chang L, Ernst T. fMRI-acoustic noise alters brain activation during working memory tasks. NeuroImage. 2005; 27:377-386. [PubMed: 15893942]

36. Benjamin C, et al. The influence of rest period instructions on the default mode network. Front Hum Neurosci. 2010; 4:218.10.3389/fnhum.2010.00218 [PubMed: 21151779]

37. Gaab N, Gaser C, Zaehle T, et al. Functional anatomy of pitch memory-an fMRI study with sparse temporal sampling. NeuroImage. 2003; 19:1417-1426. [PubMed: 12948699]

38. Keil B, et al. Size-optimized 32-channel brain arrays for $3 \mathrm{~T}$ pediatric imaging. Magn Reson Med. 2011; 66:1777-1787.10.1002/mrm.22961 [PubMed: 21656548]

39. Brown TT, et al. Prospective motion correction of high-resolution magnetic resonance imaging data in children. NeuroImage. 2010; 53:139-145. S1053-8119(10)00861-X [pii]. 10.1016/ j.neuroimage.2010.06.017 [PubMed: 20542120]

40. Fonov V, et al. Unbiased average age-appropriate atlases for pediatric studies. NeuroImage. 2011; 54:313-327. S1053-8119(10)01006-2 [pii]. 10.1016/j.neuroimage. 2010.07.033 [PubMed: 20656036]

41. Sanchez CE, Richards JE, Almli CR. Neurodevelopmental MRI brain templates for children from 2 weeks to 4 years of age. Dev Psychobiol. 2012; 54:77-91.10.1002/dev.20579 [PubMed: 21688258]

42. Ghosh SS, et al. Evaluating the validity of volume-based and surface-based brain image registration for developmental cognitive neuroscience studies in children 4 to 11 years of age. NeuroImage. 2010; 53:85-93. S1053-8119(10)00827-X [pii]. 10.1016/j.neuroimage.2010.05. 075 [PubMed: 20621657]

43. Kuklisova-Murgasova M, et al. A dynamic 4D probabilistic atlas of the developing brain. NeuroImage. 2011; 54:2750-2763. S1053-8119(10)01311-X [pii]. 10.1016/j.neuroimage. 2010.10.019 [PubMed: 20969966]

44. Raschle NM, Lee M, Buechler R, et al. Making MR imaging child's play-pediatric neuroimaging protocol, guidelines and procedure. J Vis Exp. 2009; 29:1309. [PubMed: 19684560]

45. Armstrong TS, Aitken HL. The developing role of play preparation in paediatric anaesthesia. Paediatr Anaesth. 2000; 10:1-4. pan406 [pii]. [PubMed: 10632902]

46. Hinton VJ. Ethics of neuroimaging in pediatric development. Brain Cogn. 2002; 50:455-468. S0278262602005213 [pii]. [PubMed: 12480490]

47. Fenton A, Meynell L, Baylis F. Ethical challenges and interpretive difficulties with non-clinical applications of pediatric FMRI. Am J Bioeth. 2009; 9:3-13. 907480516 [pii]. 10. 1080/15265160802617829 [PubMed: 19132609]

48. Thomason ME. Children in non-clinical functional magnetic resonance imaging (FMRI) studies give the scan experience a "thumbs up". Am J Bioeth. 2009; 9:25-27. 907480617 [pii]. 10. 1080/15265160802617928 [PubMed: 19132616]

49. Connors CM, Singh I. What we should really worry about in pediatric functional magnetic resonance imaging (FMRI). Am J Bioeth. 2009; 9:16-18. 907480817 [pii]. 10.1080/15265160802617944 [PubMed: 19132612] 